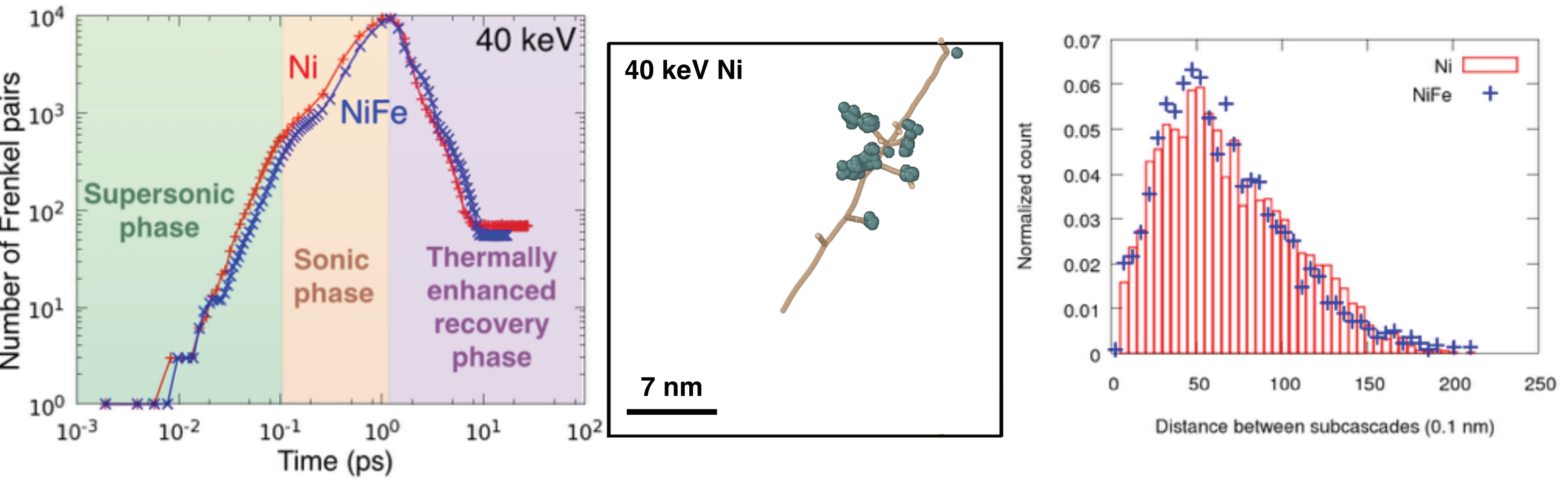




\title{
The effect of alloying nickel with iron on the supersonic ballistic stage of high energy displacement cascades
}

\author{
Laurent Karim Béland*, Yuri N. Osetsky, Roger E. Stoller \\ Materials Science and Technology Division, Oak Ridge National Laboratory, Oak Ridge, Tennessee 37831-6138, USA
}

\begin{abstract}
Previous experimental and theoretical studies suggest that the production of extended defect structures by collision cascades is inhibited in equiatomic NiFe, in comparison to pure Ni. It is also known that the production of such extend defect structures results from the formation of subcascades by high-energy recoils and their subsequent interaction. A detailed analysis of the ballistics of $40 \mathrm{keV}$ cascades in $\mathrm{Ni}$ and $\mathrm{NiFe}$ is performed to identify the formation of such subcascades and to assess their spatial distribution. It is found that subcascades in Ni and NiFe are created with nearly identical energies and distributed similarly in space. This suggests that the differences in production of extended defect structures is not related to processes taking place in the ballistic phase of the collision cascade. These results can be generalized to other, more chemically complex, concentrated alloys where the elements have similar atomic numbers, such as many high-entropy alloys.
\end{abstract}

Keywords: radiation damage, Nickel, Nickel-Iron, molecular dynamics, high-entropy alloys

\footnotetext{
Copyright notice: This manuscript has been authored by UT-Battelle, LLC under Contract No. DE-AC05-00OR22725 with the U.S. Department of Energy. The United States Government retains and the publisher, by accepting the article for publication, acknowledges that the United States Government retains a nonexclusive, paid-up, irrevocable, world-wide license to publish or reproduce the published form of this manuscript, or allow others to do so, for United States Government purposes. The Department of Energy will provide public access to these results of federally sponsored research in accordance with the DOE Public Access Plan (http://energy.gov/downloads/doe-public-access-plan).
}

\section{Introduction}

The study of radiation damage in Ni-based alloys is relevant because they act as a good model system for the austenitic stainless steels that are broadly used nuclear power applications. While it is well known that the microstructure of post-irradiation alloys critically depends on composition, many of the physical underpinnings of these different responses are still a mystery, which often leads to somewhat ad hoc material design rules. Recently, concentrated single-phase alloys, also known as high-entropy alloys, were proposed as potentially radiation-tolerant materials $[1,2]$. These alloys possess a simple face centered cubic structure (fcc), but are made of several principle elements (such as $\mathrm{NiFeCoCrMn}$ ), in contrast with conventional alloys that typically contain only one principle component. This high chemical complexity was shown to reduce electronic $[2,3]$ and phonon [4] mean free paths,

\footnotetext{
* Corresponding author

Email address: belandlk@ornl.gov (Laurent Karim Béland )
}

as well as slow down defect mobility $[5,6]$. While these properties are known to be important for radiation damage evolution, the mechanisms that explain energy dissipation and defect production in these systems remain largely unexplored.

An important aspect of radiation response and damage accumulation is related to primary damage production. Investigation of such damage production is typically done by simulating the collision cascades produced by primary knock-ons atoms (PKAs) of high-energy particules. Such molecular dynamics studies are now practically routine. Their results are usually characterized in three main dimensions: number of defects produced, distribution defect cluster (size, composition and number) and order/disorder transitions. In the case of concentrated alloys, notable investigations have focused on $\mathrm{NiAl}[7,8,9]$ and $\mathrm{NiAg}$ [10] alloys, which tend to amorphize under such conditions, $\mathrm{CuAu}[10]$, and $\mathrm{Fe}_{90} \mathrm{Cr}_{10}[11,12,13]$, which is of interest for next-generation power plants. The latter case has shown little differences in defect production and defect cluster size. In contrast with the $\mathrm{FeCr}$ case, recent studies in concentrated solid solutions (NiFe $[13,14,2,15,16]$, $\mathrm{NiCr}[16]$, NiCo [2, 15], and $\mathrm{NiCoCr}$ [17]) indicate that such alloying has an important effect on both the number of stable defect produced and the distribution of defect clusters. Understanding the physical processes dictating the radiation response in these materials is an important step towards a better understanding of the high-entropy alloys that are composed of the same elements as these binary and ternary alloys.

For example, in the case of equiatomic NiFe and $\mathrm{NiCo}$ 


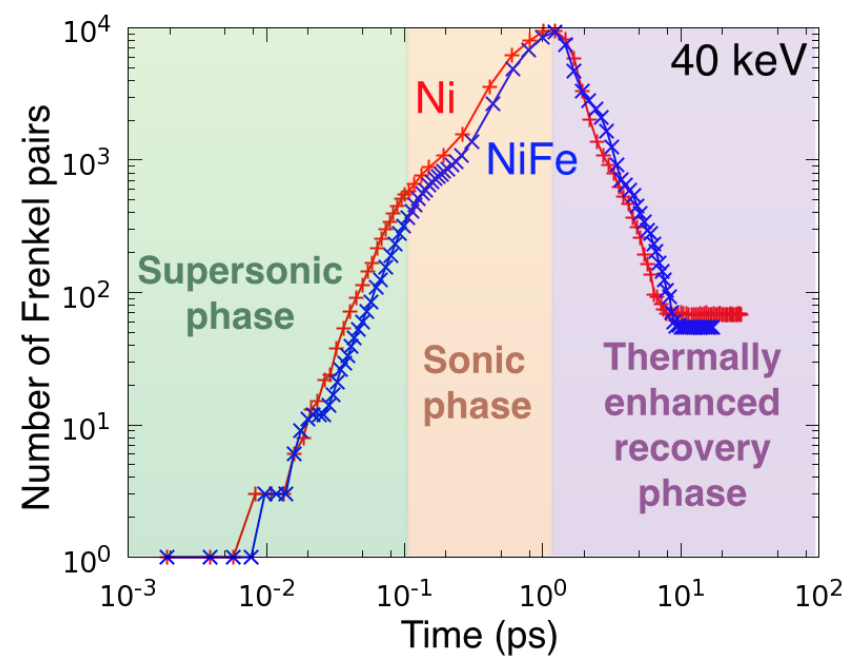

Figure 1: Typical time profiles of the number of defects during $40 \mathrm{keV}$ cascades in $\mathrm{Ni}$ and $\mathrm{NiFe}$. The three stages of the cascade [19] are illustrated by the background color. Notice the change in the log-log slope at 0.1 ps.

alloys [15], it was found that, for PKA energy of $20 \mathrm{keV}$ or less, damage reduction can be explained by the energetics of short-range interatomic interactions and by reduced thermal conductivity $[4,2]$, in comparison to pure Ni. Stiffer short-range interactions in the alloys, relative to pure $\mathrm{Ni}$, lead to less damage formation during the supersonic stage of the collision cascade. Low thermal conductivity in the alloys, relative to pure $\mathrm{Ni}$, extends the lifetime of the thermally enhanced recovery stage, which favors the self-annihilation of defects. However, it was found that these mechanisms cannot explain primary damage reduction at higher PKA energies, e.g. at $40 \mathrm{keV}$. These higher energies are of particular importance since they directly generate extended defect structures such as stacking fault tetrahedra and dislocations loops that are difficult to explain by classical nucleation mechanisms driven by point defect aggregation. These larger defects are significant because of their prominent role in radiation-induced microstructural evolution and mechanical property changes [18].

In this article, the differences in primary defect production in $\mathrm{Ni}$ and $\mathrm{NiFe}$ at $40 \mathrm{keV}$ is investigated in further detail. A review of the current state of knowledge is presented, which highlights the importance of the formation and interactions of subcascades. Such subcascades are then identified and characterized in both materials. A careful comparison is performed and discussed.

\section{A brief review}

\subsection{Three phases of collision cascade}

Collision cascades have historically been divided in two stages, the ballistic stage and a thermally enhanced recovery stage [20]. More recently, a careful analysis indicated that the ballistic stage can be further split into a supersonic and a sonic phase [19]. These can be identified by a change in slope of a logarithmic time-profile of the number of Frenkel pairs $\left(N_{F P}\right)$ during a cascade, as illustrated in Fig. 1.

High-energy recoils involving kinetic energies larger than the displacement threshold take place during the first stage, i.e. the supersonic phase. As evidenced in Refs. [19] [21], these recoils lead to concerted motion of atoms. Low-density pockets of atoms are formed, surrounded by a high-density, supersonic pressure wave, involving pressures of the order of tens of GPa. A few hypersonic recoils will escape the pressure wave, eventually creating their own low-pressure pockets (i.e. subcascades). The supersonic shockwave creates permanent defects along its trajectory, given the high kinetic energies and pressures involved. It was also shown that the interaction of these subcascades leads to the formation of extended defects (see subsection 2.3 for more details).

As the supersonic shockwave propagates and is attenuated, the kinetic energy of the fastest atoms in the system will become lower than the displacement threshold energy. This corresponds to the moment the shockwave enters the sonic regime. In other words, the atoms no longer have enough energy to knock their neighbors permanently out of position after this point in time. In that sense, the supersonic shockwave is a destructive wave, while the sonic wave is non-destructive. In a previous account of cascades in $\mathrm{Ni}$ and Ni-alloys [15], the $N_{F P}$ at this supersonic-sonic transition point (or transonic $N_{F P}$ ) was averaged over several dozens of runs. Naturally, this quantity is roughly proportional to cascade energy $E^{\text {cascade }}$. More interestingly, it was found that each material had its own characteristic transonic $N_{F P} / E^{\text {cascade }}$. Furthermore, the average number of stable FP found in a given material and a given PKA energy is proportional to its average transonic $N_{F P}$. This relationship is especially strong for PKA energy lesser than $20 \mathrm{keV}$. This evidence is consistent with the idea that permanent displacements are created during the supersonic stage and that they are no longer created after the supersonic-sonic transition.

It should also be noted that the relationship between transonic $N_{F P}$ and the stable $N_{F P}$ breaks down for PKA energies larger than $20 \mathrm{keV}[15,22]$. This observation is unexplained and is the focus of the current study. It is known that at these energies, fragmentation and subcascade formation becomes important. This is a complex fractal process, that has profound consequences on primary damage production [23, 24, 25].

The second stage corresponds to the sonic shock propagation. While no new permanent defects are created, many elastic displacements are created, which register as a large spike in the number of FP, as identified by atomic displacements or Wigner-Seitz analysis (e.g. around 1 ps in Fig. 1). This process is reversible and does not increase the number of miscoordinated atoms. The time-profile of $N_{F P}$ can take different shapes in the sonic phase, that depend 
on the details of the classical potential at hand $[20,15]$. Once again, the displacements caused by this sonic shockwave are transient, corresponding to elastic shifts of atoms that eventually recover their original positions. Nonetheless, these elastic shockwaves may have other effects. They may interact with the preexisting microstructure and influence its evolution. Likewise, they may displace defect precursors created by the supersonic shockwave and thus influence extended defect formation. Also, during the sonic stage, which lasts nearly one picosecond, atoms and defect precursors inside the sphere formed by the shockfront evolve rapidly, given the high temperatures (thousands of Kelvins) near the center of the subcascades. This leads to both aggregation and recombination of defects.

A rapid decrease of $N_{F P}$ is observed during the third stage, i.e. the thermally enhanced recovery. The system recovers from the elastic displacements created during the sonic stage and the cores of the subcascades cool down and densify. The center of the subcascades core, which are initially in a liquid-like state (i.e. are miscoordinated), recrystallize. Depending on thermal conductivities, temperatures at the center of the subcascade cores remain over $1000 \mathrm{~K}$ for a few ps to more than $10 \mathrm{ps}$. This can be especially long in concentrated alloys, where lattice and electronic thermal conductivities can be quite low $[4,2,15,26]$. At these elevated temperatures, coarsening and annhihilation of defects and defect precursors is quite important. This influences both the magnitude of defect production and the nature of the defects.

\subsection{The supersonic to sonic transition and the importance of short-range interatomic interactions}

In another study [21], it was found that the transonic $N_{F P} / E^{\text {cascade }}$ is determined by the interactions at interatomic distances between 1.4 and $2 \AA$ in Ni and concentrated Ni-alloys, which in turns largely determines final defect production. Primary defect production is dependent on the strength of interatomic interactions as close as $50 \%$ of the equilibrium interatomic distance. In order to adequately predict primary damage production, one should use interatomic potentials that adequately describe interactions at these distances (i.e. between 1.4 and $2 \AA$ ). In current practice, while interactions at very short distances (less than $1 \AA$ ) are described by the well-accepted Ziegler-Biersack-Littmark (ZBL) [27], the interaction energies at intermediate distances (1.4 and $2 \AA$ ) are usually interpolated to equilibrium distance interaction energies in rather ad hoc fashion. While this is of relatively little importance for qualitative or semi-quantitative assessments of radiation damage, it is crucial for detailed quantitative comparisons. See Ref. [28] for an more complete explanation of this issue and an example of a framework to resolve it. It is worth noting that these correspond to local pressures of the order of several tens of GPa, comparable to what is found in laser ablation and high-velocity ballistic impact simulations.

\subsection{Formation of extended defects}

The defect yield per amount of energy deposited by the PKA $\left(N_{F P} / E^{\text {cascade }}\right)$ depends non-monotonically on PKA energy [15]. It reaches a minimum around $10 \mathrm{keV}$, and then increases with PKA energy. In other words, per unit of deposited energy, the high-energy cascades are more effective at creating defects. Likewise, as the PKA energy increases from $10 \mathrm{keV}$ to $40 \mathrm{keV}$, a larger proportion of defects are generated as clusters, rather than as point defects. Undoubtedly, these two phenomena are related.

After $40 \mathrm{keV}$ cascades, smaller and fewer defect clusters are found in $\mathrm{NiFe}$ and $\mathrm{NiCo}$ than in pure Ni. The $N_{F P} / E^{\text {cascade }}$, while comparable in $\mathrm{Ni}$ and its alloys between 1 and $20 \mathrm{keV}$, is much lower in the alloys at $40 \mathrm{keV}$. It is worth noting that the displacement threshold energies are nearly identical in $\mathrm{Ni}$ and its alloys, and is therefore not related to this effect. Also, in a NiCo alloy where interatomic interactions were artificially softened between 1.4 $\AA$ and $2 \AA, 40 \mathrm{keV}$ cascades generated smaller and fewer extended defect clusters than in pure $\mathrm{Ni}$, although a larger transonic $N_{F P}$ was observed in this alloy than in pure Ni. Using the same softened NiCo alloy led to a larger number of stable defects in lower energy cascades.

It is well known that $40 \mathrm{keV}$ PKAs lead to subcascade formation. Careful analysis of such high-energy events indicate that extended defect formation is directly associated to subcascades and their interactions [19, 29, 24]. The idea is that each subcascade becomes the center of its own spherical supersonic shockwave, which temporarily creates a low-density zone surrounded by a high-density layer of atoms. The displacement and interactions of these density fluctuations can lead to "trapping" of these fluctuations. These trapped density fluctuations serve as precursors, that become extended defects as the system cools down and recovers from the supersonic and sonic displacements.

\section{Methodology}

A question naturally arises from the brief review presented in Sec. 2: "Are there significant differences in the way subcascades are formed in concentrated Ni-alloys, in comparison to Ni?"

In this article, $\mathrm{NiFe}$ was chosen as an example, but our conclusions can probably be generalized to many other alloys that serve as subcomponents of Ni-containing highentropy alloys, notably FeNiCrCoMn, as these alloys possess similar atomic mass, atomic numbers, and equilibrium properties.

\subsection{Molecular dynamics setup}

In order to gain statistically significant insights into the the formation of the subcascades, 64 cascades of $40 \mathrm{keV}$ initial kinetic energy were simulated using molecular dynamics (MD). The LAMMPS [30] software package was used. The FeNiCr potential [31] published by Bonny et 
al. in 2013, designed to model radiation-induced defects, was chosen to describe the Ni-Ni, Ni-Fe and Fe-Fe interactions. We used the version of the potential distributed in the NIST database [32]. A variable timestep was employed to limit displacements between integration steps. The simulation cell was a $80 \times 80 \times 80$ lattice-parameter cube, containing 2048000 atoms. In $\mathrm{NiFe}$, chemical composition of each site is chosen at random. All PKAs were Ni. All PKAs had initial velocity in the [135] direction, to limit channeling. The system was initially equilibrated at $300 \mathrm{~K}$ and zero pressure, using a different initial random velocity distributions in each run. The simulations were performed at constant NVE, using the Verlet-Velocity algorithm.

It is very well known that the choice of classical potential profoundly affects the results of a MD simulation. In the case of alloys, particularly those with complex phase diagrams, such as NiFe, careful care must be taken when designing and validating the potential if one desires quantitatively accurate results. Furthermore, in the case of collision cascades, it was shown that the transonic $N_{F P}$ and, consequently, the final number of stable defects, depends on interatomic interaction at short distance (in the case of $\mathrm{Ni}$, between $1.4 \AA$ and $2 \AA$ ) [21]. In our study, such validation and care was limited. The potential at hand accurately reproduces most equilibrium properties of the pure materials, stabilizes the same defect structures as those observed in experiments, has adequate binding energies between point defects and solute atoms and converges to the well-accepted Ziegler-Biersack-Littmark (ZBL) [27] values at very short distances (less than $1 \AA$ ) [28]. However, the formation and diffusion energies of defects, especially in $50 \% \mathrm{NiFe}$, do not match ab initio data. Likewise, the interaction between $1.4 \AA$ and $2 \AA$ between $\mathrm{Ni}-\mathrm{Ni}$, $\mathrm{Ni}-\mathrm{Fe}$ and $\mathrm{Fe}-\mathrm{Fe}$ pairs are much stiffer than those predicted by $a b$ initio data. It should not be seen as an appropriate potential to make a precise quantitative assessment of primary damage production in $\mathrm{Ni}$ and $\mathrm{NiFe}$, but rather as a tool to explore the phenomena describing collision cascades in face-centered cubic solid solutions containing $\mathrm{Ni}$ and other elements with similar mass and atomic number.

\subsection{Identifying subcascades}

In order to identify and characterize subcascades, a criterium was established based on atoms of very low coordination. The total number of neighbors within a distance of 1.6 lattice-parameters of each atom was calculated; this number is a coordination number. At equilibrium, 73 atoms would be found within 1.6 lattice-parameters of a given atom. We found that atoms that sit near the center of a supersonic shockwave can have coordination numbers lower than 50 at the moment of supersonic to sonic transition, defined as $0.2 \mathrm{ps}$ in our analysis. The position and magnitude of the supersonic waves created by the subcascades can be identified through these atoms with coordination number lower than 50. An illustration of the use of this criterium is presented in Fig. 2. In that figure, the small beige atoms represent all the positions occupied by

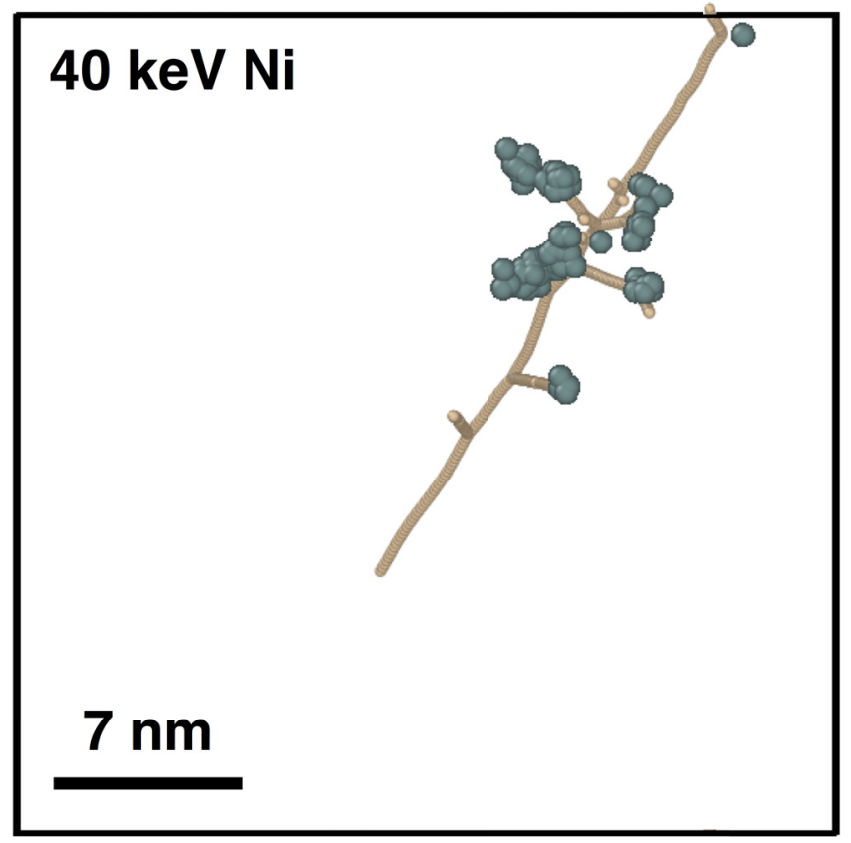

Figure 2: An illustration of a $40 \mathrm{keV}$ cascade in Ni and its subcascades. The small beige spheres are all the positions occupied by atoms with kinetic energy larger than $500 \mathrm{eV}$ during the course of a typical cascade. The "branches" represent the creation of a highly energetic secondary knock-on, i.e. a subcascade. The blue spheres are the atoms with low atomic coordination at $0.2 \mathrm{ps}$ (low atomic coordination is defined as less than 50 neighbors at a 1.6 lattice-parameter distance). The cascade was initiated in the bottom-center of the box. The high-energy trajectory crossed the periodic boundary near its end. 


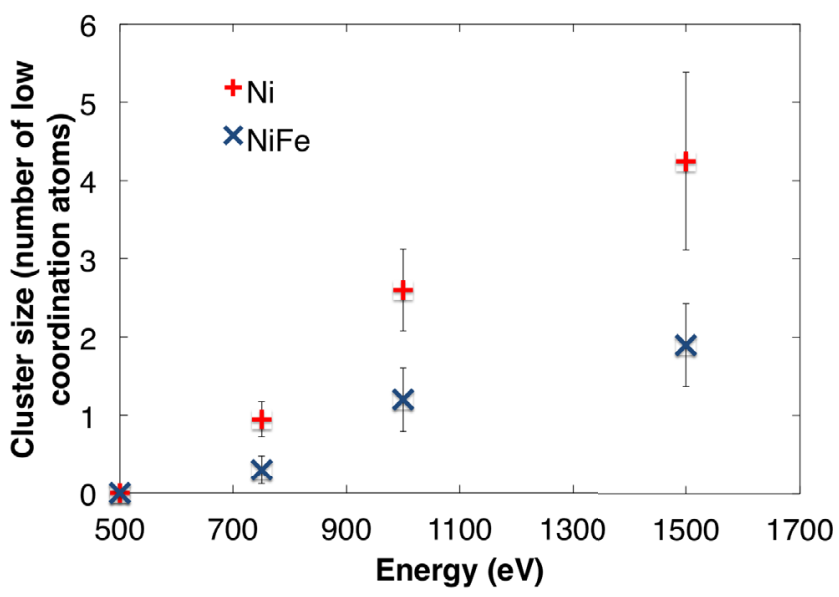

Figure 3: The size of the low-density pocket created by a supersonic shockwave induced by PKAs with initial kinetic energies between $500 \mathrm{eV}$ and $1500 \mathrm{eV}$ in $\mathrm{Ni}$ and NiFe. The size of the low-density pocket is determined by the number of atoms having fewer than 50 neighbors within 1.6 latticeparameters. The error bars correspond to the standard error.

an atom with a kinetic energy larger than $500 \mathrm{eV}$ during the cascade. The large blue spheres represent the atoms with a coordination number smaller than 50 at 0.2 ps into the cascade. A $40 \mathrm{keV}$ cascade was initiated in the bottom center of the box figure. One can easily notice that the positions of the blue spheres corresponds to the endpoint of high-energy secondary knock-ons. In other words, subcascades can be identified at the location of these blue spheres.

The relationship between the number of atoms in a lowcoordination atom cluster and the energy of a subcascade was investigated. In $\mathrm{Ni}$ and $\mathrm{NiFe}$, cascades with initial PKA kinetic energy within $500 \mathrm{eV}$ and $1500 \mathrm{eV}$, where subcascade formation is very weak, were performed. 20 cascades were performed per energy. The number of lowcoordination atoms (less than 50 neighbors at 1.6 latticeparameters) was calculated at $0.15 \mathrm{ps}$ (which corresponds to the moment of the transonic to sonic transition at 1 $\mathrm{keV}$ ). The results of these calculations are plotted in Fig. 3. There is a clear positive relationship between the number of low-coordination atoms and cascade energy. The energy threshold to create such an atom is between $500 \mathrm{eV}$ and $750 \mathrm{eV}$, both in $\mathrm{Ni}$ and NiFe. Also, low-density pockets are systematically smaller in NiFe than in Ni. Overall, it seems that the size of a low-coordination cluster is a good indicator of the presence and of the magnitude of a high-energy recoil.

\subsection{Two-point correlation function}

The two-point correlation function, as introduced by Simeone, Luneville and Serruys[23], was also computed:

$$
\left.C(\boldsymbol{r})=\frac{1}{N}\left\langle\sum_{\boldsymbol{y}} \rho(\boldsymbol{y}) \rho(\boldsymbol{r}+\boldsymbol{y})\right)\right\rangle_{\Omega(\boldsymbol{r})}
$$

where $N$ is the number of low density atoms or atomic displacements in a cascade, $\rho(\boldsymbol{y}) \rho(\boldsymbol{y}+\boldsymbol{r})$ is equal to 1 if there are low density atoms or atomic displacements at positions $\boldsymbol{y}$ and $\boldsymbol{r}+\boldsymbol{y}$, or 0 otherwise. The brackets denote an average over all solid angles in all the cascades simulated. $C(\boldsymbol{r})$ only depends on the distance between two atoms $r$. Furthermore, given the fractal nature of collision cascades $C(r)$ scales as a power law $r^{-\beta}$. This exponent is related to the fractal dimensionality $D$ of the process as $D=-\beta+3$.

This indicator was previously used to establish the dimensionality of the fractal structures observed during collision cascades. In principle, one should find the slope of the two-point correlation function in a log-log plot. However, given the finite size of cascades, such the log-log two-point correlation function actually have a pronounced curvature, leading to ambiguity as to the fitting of a power law. Nevertheless, given the past success of this method at identifying changes in subcascade and cluster formation, it was used to further enhance our characterization and comparison of the systems at hand.

\section{Results and analysis}

The similarity between the time-profiles of $\mathrm{Ni}$ and $\mathrm{NiFe}$ presented in Fig. 1 is striking. The supersonic to sonic transition and the peak in displaced atoms happen simultaneously in $\mathrm{Ni}$ and $\mathrm{NiFe}$. In this particular case, the number of displacements was slightly smaller in $\mathrm{NiFe}$ at the supersonic to sonic transition point. The recovery of atomic displacements after the peak was slightly faster in Ni. Generally, variations of the time-profile of cascades in a given material from one run to another are of the same amplitude as variations between materials. In other words, one needs to perform a statistical analysis over several tens of runs to identify physically meaningful phenomena. Nonetheless, the similarities between the time-profile of displaced atoms indicates that the same framework can be used to characterize cascades in both materials. This is not surprising, given the similarities of both materials, e.g. atomic mass, atomic number, lattice parameter, elastic constants, and displacement threshold energy [15].

32 runs in $\mathrm{Ni}$ and $\mathrm{NiFe}$ were used to calculate the secondary knock-on energy spectra. This was accomplished by periodically verifying if any new atom in the system had kinetic energy greater than $500 \mathrm{eV}$. This was considered as a knock-on event. The results of these calculations are plotted in Fig. 4 (a). There is no statistically significant difference between the $\mathrm{Ni}$ spectrum and the $\mathrm{NiFe}$ 

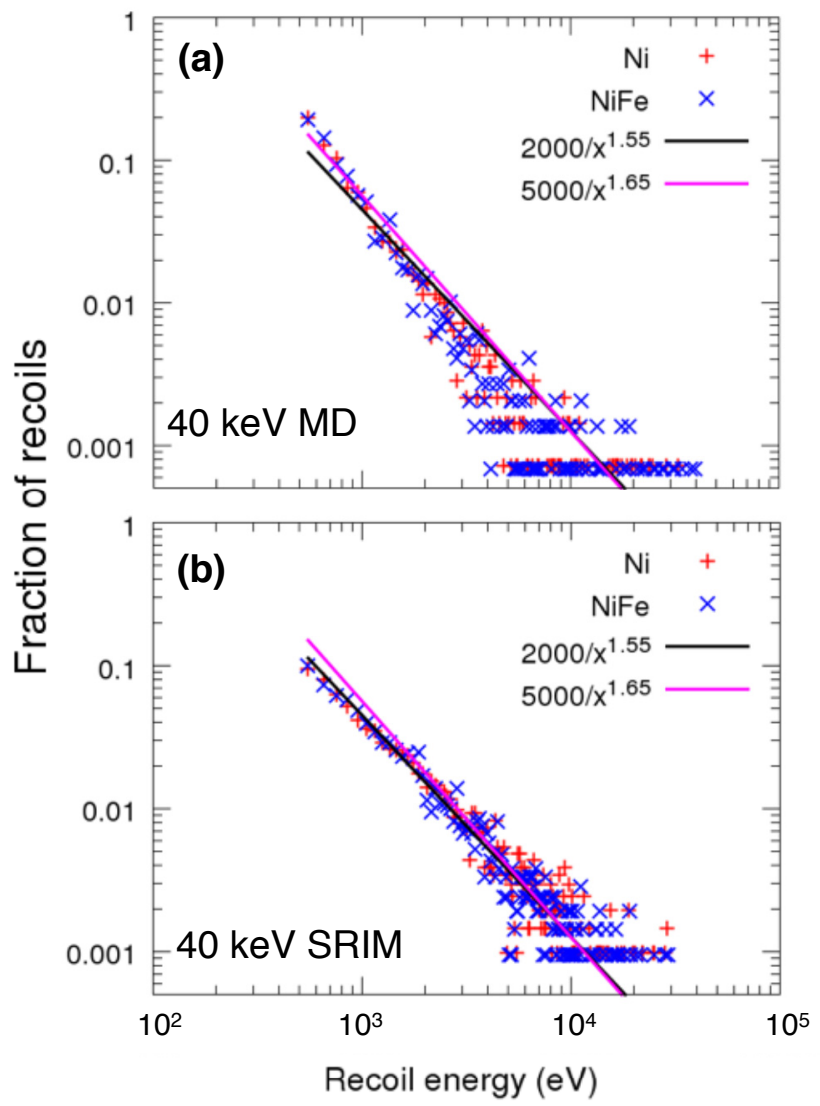

Figure 4: The normalized recoil spectra produced by 40 keV cascades in $\mathrm{Ni}$ and $\mathrm{NiFe}$ as computed using (a) MD and (b) SRIM.

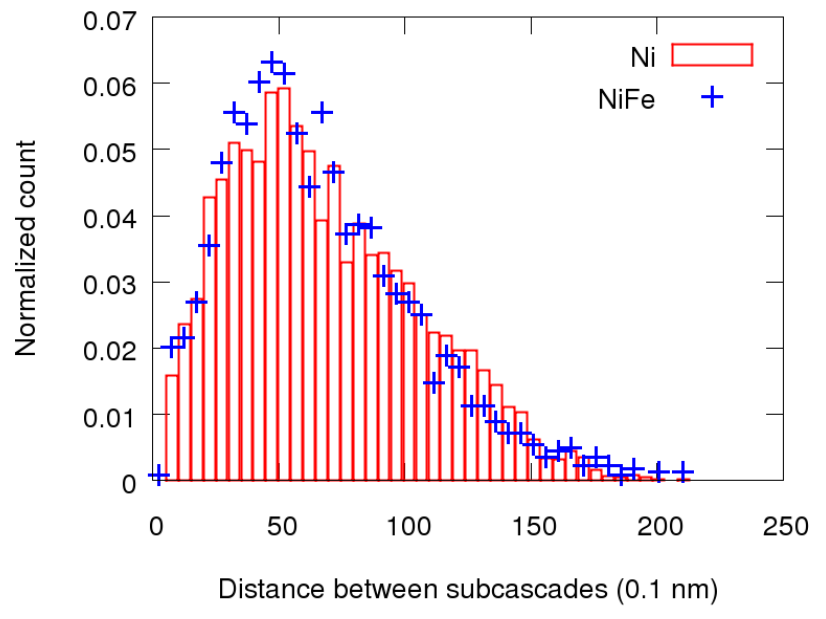

Figure 5: The distribution of center-to-center distances between subcascades in $\mathrm{Ni}$ and NiFe. For sake of readability, a bar plot is used for $\mathrm{Ni}$ and crosses are used for $\mathrm{NiFe}$.

spectrum. They are both described by a power law of coefficient -1.5. The same calculation was also performed with the Stopping Range of Ions in Matter [33] (SRIM), a Monte Carlo code based on the binary collision approximation (BCA) and the ZBL potential [27], simulating 200 cascades with $40 \mathrm{keV}$ energy in full cascade mode. The results are nearly identical to our MD results. Unsurprisingly, the BCA is very good at these high kinetic energies. It seems that the differences in masses and screened Coulomb interactions between $\mathrm{Ni}$ and $\mathrm{NiFe}$ are simply not sufficient to significantly impact the ballistic processes that generate energetic knock-ons.

The spatial distribution of the subcascades is illustrated in Fig. 5. The center-to-center distance between all clusters of atoms with low coordination is measured and binned. There are three remarkable features. First, the distances follow a distribution peaked at $5 \mathrm{~nm}$ in both $\mathrm{Ni}$ and NiFe. Second, both distributions end at $22 \mathrm{~nm}$. Third, the overall distributions found for $\mathrm{Ni}$ and $\mathrm{NiFe}$ are nearly indistinguishable. It seems that there are no significant differences in the spatial distribution of subcascades in $\mathrm{Ni}$ and NiFe.

The normalized two-point correlation functions of the low-coordination atoms and Wigner-Seitz (WZ) defects at $\mathrm{t}=0.2 \mathrm{ps}$ are plotted in Fig. 6. The trends, slopes and values in $\mathrm{Ni}$ and $\mathrm{NiFe}$ are very similar, which indicate that the fractal dimension of the processes at hand are identical. One should note that the noise in the lowcoordination atoms two-point correlation is more important than for WZ defects, because there are only a few tens of low-coordination atoms per cascade, compared to a few hundred $\mathrm{WZ}$ defects at $\mathrm{t}=0.2 \mathrm{ps}$. The power-law for the low-coordination atoms has a coefficient of about -1 , which is equivalent to a fractal dimensionality of 2 . The power law for the WZ defects has a coefficient of about 0 , which is equivalent to a fractal dimensionality of 3 . These 


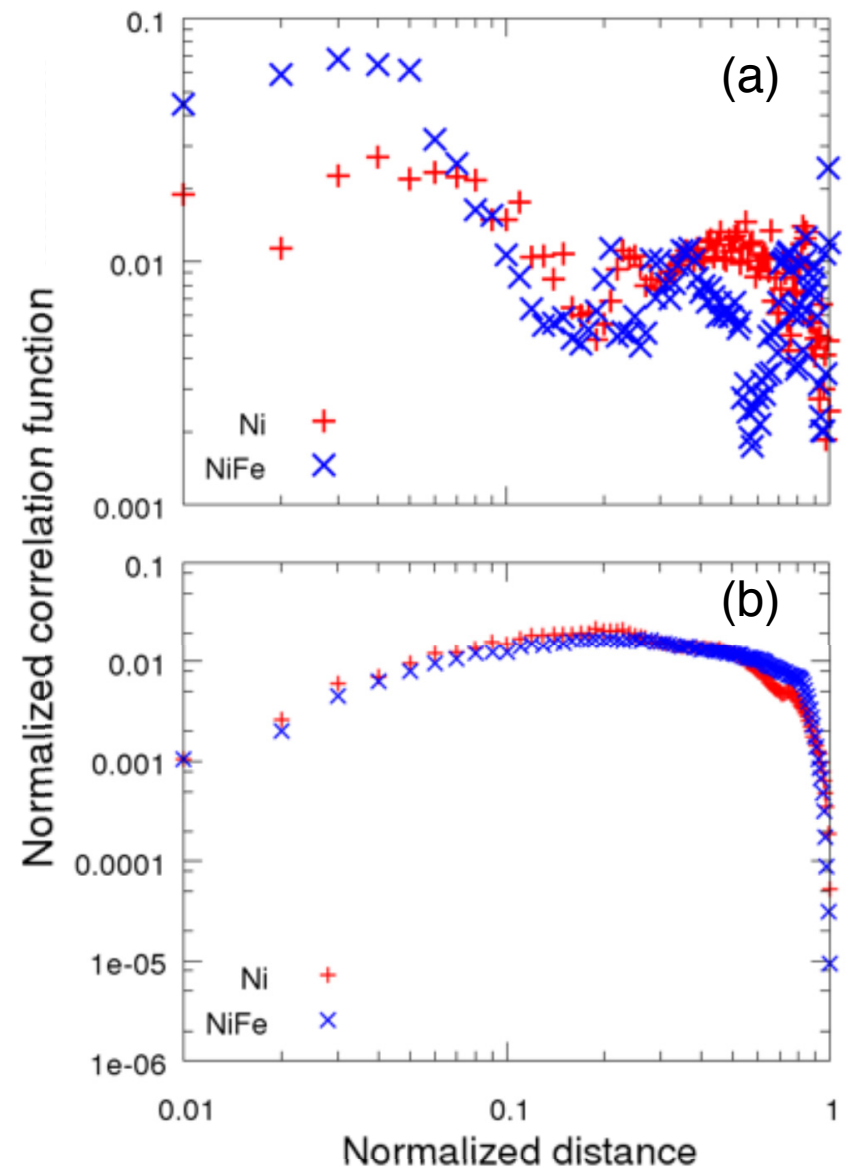

Figure 6: The two-point distribution functions of (a) low coordination atoms and (b) Wigner-Seitz defects at $\mathrm{t}=0.2$ ps in $\mathrm{Ni}$ and $\mathrm{NiFe}$.

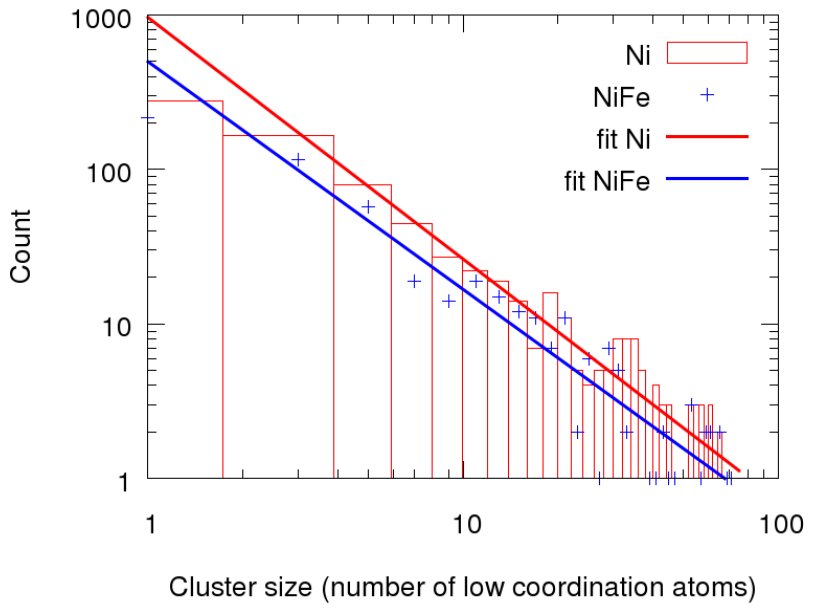

Figure 7: The size distribution of low coordination atom clusters in $\mathrm{Ni}$ and NiFe. The full lines are the best powerlaw fit to the data. The power laws have coefficients -1.47 \pm 0.1 and $-1.56 \pm 0.13$ in $\mathrm{Ni}$ and $\mathrm{NiFe}$, respectively.

coefficients are identical in $\mathrm{Ni}$ and $\mathrm{NiFe}$. However, the deviations from the trend (minima and maxima) in $\mathrm{Ni}$ and $\mathrm{NiFe}$ are slightly offset, which is consistent with different average cluster sizes in $\mathrm{Ni}$ and $\mathrm{NiFe}$.

The size distributions of the low coordination atom clusters in $\mathrm{Ni}$ and $\mathrm{NiFe}$ are plotted in Fig. 7. They are well described by power laws of coefficients $-1.47 \pm 0.1$ and $1.56 \pm 0.13$ in $\mathrm{Ni}$ and NiFe, respectively. The difference in slope between the fits is not statistically significant. For each cluster size, less NiFe clusters were observed than $\mathrm{Ni}$ clusters.

\section{Discussion}

There are many similarities in the characteristics of subcascade formation in $\mathrm{Ni}$ and NiFe. They are generated by an identical recoil spectrum (c.f. Fig. 4), they have the same length scales (c.f. Fig. 5), they are nearly identically correlated in space (c.f. Figs. $5 \& 6$ ) and their size distributions follow the same power law coefficient (c.f. 7). This indicates that the ballistic processes that lead to shockwave formation are essentially identical in both materials.

However, the total number of low coordination atoms found in $\mathrm{Ni}$ and $\mathrm{NiFe}$ at the time of the supersonic to sonic transition is quite different. In 64 runs, 6175 such low coordination atoms were seen in $\mathrm{Ni}$, against 4084 in NiFe. Likewise, 765 low density pockets were found in $\mathrm{Ni}$, against 541 in NiFe. Thus, the average low coordination atom cluster size was 8.07 in Ni, compared to 7.55 in NiFe. This can be understood by observing Figs. 3 \& 7 . A knock-on which might have produced a low coordination atom cluster of size 4 in $\mathrm{Ni}$ will only produce one of size 2 in $\mathrm{NiFe}$. Combined with the fact that the knock-on spectra are identical in the two materials, this explains why the 
power law distribution of Fig. 7 is shifted to the left. The surface area between the fit for $\mathrm{Ni}$ and NiFe in Fig. 7 corresponds to the difference in the total of number of low coordination atoms observed in our simulations.

It should be understood that the two-point correlation function analysis and the two other cluster-based analysis (center-to-center distances and cluster-size distribution) are tightly linked. The minima and maxima of the correlation functions plotted in Fig. 6(a) are linked to the probability of observing two low-density atoms at a short distance of each other (i.e. to the cluster size distribution), the probability of observing two low-density atoms apart a certain distance (i.e. to the inter-cluster distances) and the number of clusters within the region where the function is calculated. The differences and similarities in the functions plotted in Fig. 6(a) are thus directly linked and consistent with the other differences and similarities mentioned above.

Why are the pockets smaller in NiFe? This is mostly due to the stiffness of the interatomic potential at distances between $1.4 \AA$ and $2.0 \AA$. As illustrated in Ref. [21], these correspond to the shortest distances in the supersonic shockfront as it nears the transition to a sonic shockfront. The energetics at these short distances limits the size of the low-density pocket. In the case of the classical potential used in this study, these interactions are stiffer for Ni-Fe and Fe-Fe pairs than for Ni atoms. Thus, the low-density pockets are larger in Ni. However, this is not true in all alloys. For example, the 2009 Bonny potential [34] predicts that Fe-Fe interactions are softer than $\mathrm{Ni}-\mathrm{Ni}$ interactions at the same distances. Likewise, in a former study of the effects of alloying [15], a Ni-Co-like binary alloy potential was designed purposefully so that the alloying element interactions would be much softer than the Ni-Ni interactions. The transonic $N_{F P}$ (and the size of the low-density pockets) was much larger in this binary alloy than in $\mathrm{Ni}$, in agreement with our analysis. However, a similar decrease in defect and cluster production was observed at $40 \mathrm{keV}$ in this "soft" alloy than in "stiff" NiFe and "stiff" NiCo. This indicates that this defect-reduction mechanism observed in these alloys is not related to the ballistic phase of the collision cascades, but rather to processes that take place during the phase of thermally enhanced recovery.

While our results, formally speaking, directly apply only to $\mathrm{NiFe}$, and to a lesser extent, $\mathrm{NiCo}$, the conclusions are most likely generalizable to multi-component face-centered cubic solid solutions, which components have similar mass and atomic numbers (e.g. NiFeCrCoMn). Any effect of alloying on extended defect production most probably do not originate from ballistic effects, but rather from the later stages of primary damage production. If large atomic number or mass differences exists (such as in NiPd), this conclusion might not hold. This issue, however, is out of the scope of this study.

Our analysis raises a question about the origin of reduced defect cluster production in the concentrated binary alloys. A tentative answer suggests that the potential energy landscape of these materials favor recombination of interstitial and vacancy clusters rather than the formation of larger extended defects, relative to the pure material. If this is the case, one might be able to tune alloy composition to enhance such primary damage reduction at the high end of the PKA spectrum. Likewise, the kinetic evolution of the microstructure created by the cascade on longer timescales has an important effect on defect cluster size [5]. These issues certainly warrants further investigation.

\section{Conclusions}

A thorough comparison of the supersonic ballistic process involved in $40 \mathrm{keV}$ cascades in $\mathrm{Ni}$ and $\mathrm{NiFe}$ was performed. It was found that the knock-on spectra and spatial correlation of the high-energy collisions is nearly identical in $\mathrm{Ni}$ and NiFe. Stiffer interatomic interactions between $1.4 \AA$ and $2.0 \AA$ in NiFe led the supersonic shockwaves created by the subcascades to displace less atoms than in pure Ni. This effect, however, cannot explain the large differences in extended cluster formation that is observed at the end of the cascade simulations.

\section{Acknowledgments}

This work was supported as part of the Energy Dissipation to Defect Evolution (EDDE), an Energy Frontier Research Center funded by the U.S. Department of Energy, Office of Science, Basic Energy Sciences. LKB acknowledges additional support from a fellowship awarded by the Fonds Québécois de recherche Nature et Technologies. We thank Alfredo Correa and German D Samolyuk for insightful discussions.

[1] T. Egami, W. Guo, P. Rack, T. Nagase, Irradiation resistance of multicomponent alloys, Metallurgical and Materials Transactions A 45 (1) (2014) 180-183.

[2] Y. Zhang, G. M. Stocks, K. Jin, C. Lu, H. Bei, B. C. Sales, L. Wang, L. K. Béland, R. E. Stoller, G. D. Samolyuk, et al., Influence of chemical disorder on energy dissipation and defect evolution in concentrated solid solution alloys, Nature communications 6 .

[3] K. Jin, B. C. Sales, G. M. Stocks, G. D. Samolyuk, M. Daene, W. J. Weber, Y. Zhang, H. Bei, Tailoring the physical properties of ni-based single-phase equiatomic alloys by modifying the chemical complexity, Scientific reports 6 .

[4] M. Caro, L. Béland, G. Samolyuk, R. Stoller, A. Caro, Lattice thermal conductivity of multi-component alloys, Journal of Alloys and Compounds 648 (2015) 408-413.

[5] L. K. Béland, G. D. Samolyuk, R. E. Stoller, Differences in the accumulation of ion-beam damage in ni and nife explained by atomistic simulations, Journal of Alloys and Compounds 662 (2016) 415-420.

[6] D. S. Aidhy, C. Lu, K. Jin, H. Bei, Y. Zhang, L. Wang, W. J. Weber, Point defect evolution in ni, nife and nicr alloys from atomistic simulations and irradiation experiments, Acta Materialia 99 (2015) 69-76.

[7] H. Zhu, R. Averback, M. Nastasi, Molecular dynamics simulations of a 10kev cascade in $\beta$-nial, Philosophical Magazine A 71 (4) (1995) 735-758. 
[8] F. Gao, D. Bacon, Molecular dynamics study of displacement cascades in ni3al i. general features and defect production efficiency, Philosophical Magazine A 71 (1) (1995) 43-64.

[9] N. Doan, R. Vascon, Displacement cascades in metals and ordered alloys. molecular dynamics simulations, Nuclear Instruments and Methods in Physics Research Section B: Beam Interactions with Materials and Atoms 135 (1) (1998) 207-213.

[10] T. J. Colla, H. M. Urbassek, K. Nordlund, R. S. Averback, Ioninduced mixing and demixing in the immiscible ni-ag system, Physical Review B 63 (10) (2001) 104206.

[11] N. Juslin, K. Nordlund, J. Wallenius, L. Malerba, Simulation of threshold displacement energies in fecr, Nuclear Instruments and Methods in Physics Research Section B: Beam Interactions with Materials and Atoms 255 (1) (2007) 75-77.

[12] C. Björkas, K. Nordlund, L. Malerba, D. Terentyev, P. Olsson, Simulation of displacement cascades in fe $90 \mathrm{cr} 10$ using a two band model potential, Journal of Nuclear Materials 372 (2) (2008) 312-317.

[13] K. Vörtler, N. Juslin, G. Bonny, L. Malerba, K. Nordlund, The effect of prolonged irradiation on defect production and ordering in fe-cr and fe-ni alloys, Journal of Physics: Condensed Matter 23 (35) (2011) 355007.

[14] C. Wang, W. Zhang, C. Ren, P. Huai, Z. Zhu, The effect of temperature on primary defect formation in ni-fe alloy, Nuclear Instruments and Methods in Physics Research Section B: Beam Interactions with Materials and Atoms 321 (2014) 49-53.

[15] L. K. Béland, C. Lu, Y. N. Osetskiy, G. D. Samolyuk, A. Caro, L. Wang, R. E. Stoller, Features of primary damage by high energy displacement cascades in concentrated ni-based alloys, Journal of Applied Physics 119 (8) (2016) 085901.

[16] M. W. Ullah, D. S. Aidhy, Y. Zhang, W. J. Weber, Damage accumulation in ion-irradiated ni-based concentrated solidsolution alloys, Acta Materialia 109 (2016) 17-22.

[17] F. Granberg, K. Nordlund, M. W. Ullah, K. Jin, C. Lu, H. Bei, L. M. Wang, F. Djurabekova, W. J. Weber, Y. Zhang, Improved radiation resistance in equiatomic multicomponent single phase alloys, Physical Review Letters Accepted manuscript.

[18] S. Zinkle, 1.03-radiation-induced effects on microstructure, Comprehensive Nuclear Materials, Elsevier, Oxford (2012) 6598.

[19] A. Calder, D. J. Bacon, A. V. Barashev, Y. N. Osetsky, On the origin of large interstitial clusters in displacement cascades, Philosophical Magazine 90 (7-8) (2010) 863-884.

[20] R. Stoller, 1.11 - primary radiation damage formation, in: R. J. Konings (Ed.), Comprehensive Nuclear Materials, Elsevier, Oxford, 2012, pp. 293 - 332. doi:http://dx.doi.org/10.1016/ B978-0-08-056033-5.00027-6.

URL http://www.sciencedirect.com/science/article/pii/ B9780080560335000276

[21] L. K. Béland, Y. N. Osetsky, R. E. Stoller, Atomistic material behavior at extreme pressures, NPJ Computational Materials In press,doi:10.1038/npjcompumats.2016.7.

[22] A. Sand, J. Dequeker, C. Becquart, C. Domain, K. Nordlund, Non-equilibrium properties of interatomic potentials in cascade simulations in tungsten, Journal of Nuclear Materials 470 (2016) 119-127.

[23] D. Simeone, L. Luneville, Y. Serruys, Cascade fragmentation under ion beam irradiation: A fractal approach, Physical Review E 82 (1) (2010) 011122.

[24] E. Antoshchenkova, L. Luneville, D. Simeone, R. Stoller, M. Hayoun, Fragmentation of displacement cascades into subcascades: A molecular dynamics study, Journal of Nuclear Materials 458 (2015) 168-175.

[25] W. Setyawan, A. P. Selby, N. Juslin, R. E. Stoller, B. D. Wirth, R. J. Kurtz, Cascade morphology transition in bcc metals, Journal of Physics: Condensed Matter 27 (22) (2015) 225402.

[26] G. Samolyuk, L. Béland, G. Stocks, R. Stoller, Electron-phonon coupling in ni-based binary alloys with application to displacement cascade modeling, Journal of Physics: Condensed Matter 28 (17) (2016) 175501.

[27] J. F. Ziegler, J. Biersack, U. Littmark, The stopping and range of ions in matter, vol. 1 (1985).

[28] R. E. Stoller, A. Tamm, L. K. Béland, G. D. Samolyuk, G. M. Stocks, A. Caro, L. V. Slipchenko, Y. N. Osetsky, A. Aabloo, M. Klintenberg, et al., The impact of short-range forces on defect production from high-energy collisions, Journal of chemical theory and computation Online,doi:10.1021/acs.jctc.5b01194.

[29] A. Korchuganov, K. Zolnikov, D. Kryzhevich, V. Chernov, S. Psakhie, Generation of shock waves in iron under irradiation, NIMB: Beam Interactions with Materials and Atoms (2014) in press.

[30] S. Plimpton, Fast parallel algorithms for short-range molecular dynamics, Journal of computational physics 117 (1) (1995) 119.

[31] G. Bonny, N. Castin, D. Terentyev, Interatomic potential for studying ageing under irradiation in stainless steels: the fenicr model alloy, Modelling and Simulation in Materials Science and Engineering 21 (8) (2013) 085004.

[32] C. A. Becker, Atomistic simulations for engineering: potentials and challenges, Models, Databases and Simulation Tools Needed for Realization of Integrated Computational Mat. Eng.(ICME 2010) (2014) 91 http://www.ctcms.nist.gov/potentials.

[33] J. F. Ziegler, M. D. Ziegler, J. P. Biersack, Srim-the stopping and range of ions in matter (2010), Nuclear Instruments and Methods in Physics Research Section B: Beam Interactions with Materials and Atoms 268 (11) (2010) 1818-1823.

[34] G. Bonny, R. Pasianot, L. Malerba, Fe-ni many-body potential for metallurgical applications, Modelling and Simulation in Materials Science and Engineering 17 (2) (2009) 025010. 